\title{
Nobiletin, a Polymethoxy Flavonoid, Protects Against Cardiac Hypertrophy Induced by Pressure-Overload via Inhibition of NAPDH Oxidases and Endoplasmic Reticulum Stress
}

\author{
Ning Zhanga,b Wen-Ying Wei ${ }^{a, b}$ Zheng Yang ${ }^{a, b}$ Yan Che ${ }^{a, b}$ Ya-Ge Jin ${ }^{a, b}$ \\ Hai-Han Liao ${ }^{a, b}$ Sha-sha Wang ${ }^{a, b}$ Wei Deng ${ }^{a, b}$ Qi-Zhu Tang ${ }^{a, b}$ \\ aDepartment of Cardiology, Renmin Hospital of Wuhan University, Wuhan, ${ }^{b}$ Cardiovascular Research \\ Institute of Wuhan University, Wuhan, China
}

\section{Key Words}

Nobiletin • Cardiac hypertrophy $• \mathrm{NAPDH}$ oxidase $\bullet$ Endoplasmic reticulum stress

\begin{abstract}
Background/Aims: An increase in oxidative stress has been implicated in the pathophysiology of pressure-overload induced cardiac hypertrophy. Nobiletin (NOB), extracted from the fruit peel of citrus, possesses anti-oxidative property. Our study aimed to investigate the protective role of NOB in the progression of cardiac hypertrophy in vivo and in vitro. Methods: Mice received aortic banding $(A B)$ operation to induce cardiac hypertrophy. Experimental groups were as follows: sham+vehicle $(\mathrm{VEH} / \mathrm{SH})$, sham+NOB $(\mathrm{NOB} / \mathrm{SH}), \mathrm{AB}+$ vehicle $(\mathrm{VEH} / \mathrm{AB})$, and $A B+N O B(N O B / A B)$. Animals ( $n=15$ per group) were treated with vehicle or $N O B(50 \mathrm{mg} / \mathrm{kg}$ ) for 4 weeks after disease onset. Results: NOB prevented cardiac hypertrophy induced by aortic banding $(A B)$, as assessed by the cross-sectional area of cardiomyocytes, heart weight-to-body weight ratio, gene expression of hypertrophic markers and cardiac function. In addition, NOB supplementation blunted the increased expression of NAPDH oxidase (NOX) 2 and NOX4 and mitigated endoplasmic reticulum (ER) stress and myocyte apoptosis in cardiac hypertrophy. Furthermore, NOB treatment attenuated the neonatal rat cardiomyocyte (NRCM) hypertrophic response stimulated by phenylephrine (PE) and alleviated ER stress. However, our data showed that NOB dramatically inhibited NOX2 expression but not NOX4 in vitro. Finally, we found that knockdown of NOX2 attenuated ER stress in NRCMs stimulated by PE. Conclusions: Inhibition of oxidative and ER stress by NOB in the myocardium may represent a potential therapy for cardiac hypertrophy. Moreover, there is a direct role of NOX2 in regulating ER stress stimulated by PE.




\section{Introduction}

Pathological cardiac hypertrophy is associated with a complicated spectrum of pathophysiological changes, including oxidative stress, metabolic dysfunction, sarcomere disorganization, endoplasmic reticulum (ER) stress, cell apoptosis and fibrosis [1]. Extensive study has suggested that oxidative stress plays a predominant role in the progression of cardiac hypertrophy [2]. Increased reactive oxygen species (ROS) results in cellular damage, energetic deficit and cardiac dysfunction in heart failure. A large body of evidence demonstrates that ROS generated by NADPH oxidases (NOX) are a major source in cardiac hypertrophy $[3,4]$. In addition, endoplasmic reticulum (ER) stress has been shown to increase in cardiac hypertrophy in a murine pressure-overload model [5]. ER and oxidative stress have close associations in ROS generation [6], and increased ER stress can initiate myocyte apoptosis [7]. NADPH oxidase activation contributes to ER stress and myocardial dysfunction in diabetic mice [8]. Thus, inhibition of oxidative stress is considered a potential therapy for cardiac hypertrophy [9].

There is some evidence, which indicates that nobiletin (NOB) is a flavonoid with antioxidative and anti-diabetic properties [10]. Several studies have demonstrated that NOB relieves age-related cognitive impairment, accompanied by alleviation of oxidative stress in SAMP8 mice [11]. Our previous study indicated that NOB inhibits NADPH oxidases in streptozotocin-induced diabetic cardiomyopathy [12]. Previous studies have shown that NOB inhibits LPS-induced ROS production in RAW 264.7 cells [13]. Additional studies have found that NOB can ameliorate apoptosis in SK-N-SH human neuroblastoma cells and repress TXNIP expression, which plays a crucial role in irremediable ER stress. However, there is still a paucity of data regarding the beneficial role of NOB in cardiac hypertrophy induced by aortic banding $(\mathrm{AB})$. Moreover, it remains unclear whether NOB treatment mitigates ER stress via a reduction of oxidative stress in a murine cardiac pressure-overload model. In the present study, we aimed to clarify the protective role of NOB in cardiac hypertrophy.

\section{Materials and Methods}

\section{Animals and experimental design}

All animal care and experiments were performed according to the Guidelines for the Care and Use of Laboratory Animals published by the United States National Institutes of Health (NIH Publication, revised 2011) and the institutional guidelines of the Animal Care and Use Committee of Renmin Hospital of Wuhan University (Wuhan, China). Animals were housed under specific-pathogen-free (SPF) conditions in a 12-h light and 12 -h dark cycle with food and water provided ad libitum. The AB operation and subsequent analyses were performed in a blind manner for all groups. Male C57BL/6 mice (8 to 10 weeks old; body weight: $25.5 \pm$ $2 \mathrm{~g}$ ) were purchased from the Institute of Laboratory Animal Science, Chinese Academy of Medical Sciences (Beijing, China). NOB was purchased from Shanghai Winherb Medical Science Co. Ltd. (Shanghai, China), and the purity of NOB was $>98 \%$ as determined by high-performance liquid chromatography (HPLC) analysis. The animals were randomly divided into four groups: sham+vehicle $(\mathrm{VEH} / \mathrm{SH})(\mathrm{n}=15)$, sham+NOB (NOB/ $\mathrm{SH})(\mathrm{n}=15), A B+$ vehicle $(\mathrm{VEH} / \mathrm{AB})(\mathrm{n}=15)$, and $\mathrm{AB}+\mathrm{NOB}(\mathrm{NOB} / \mathrm{AB})(\mathrm{n}=15)$. The mice were anesthetized with $3 \%$ sodium pentobarbital by intraperitoneal injection, and cardiac hypertrophy model was established via $A B$ surgery as previously described $[14,15]$. Three days after $A B$ or sham procedure, mice were treated with NOB or vehicle for 4 weeks by gavage, and the dose of NOB (dissolved in normal saline including $0.05 \%$ Tween-80) was $50 \mathrm{mg} / \mathrm{kg}$ as previously described [12]. After administration of NOB or vehicle for four weeks, all experimental mice were anesthetized with $1.5 \%$ isoflurane, euthanized by cervical dislocation, and the heart was then collected and the tibia measured for length.

Echocardiography measurement and invasive hemodynamic pressure-volume analysis

Cardiac function was detected using an echocardiographic system equipped with a 10-MHz transducer (Esaote SpA, Genoa, Italy). Briefly, lightly anesthetized mice $\left(1.5 \%\right.$ isoflurane and $98.5 \% \mathrm{O}_{2}$ ) were placed on a temperature-controlled warming pad $\left(37^{\circ} \mathrm{C}\right)$. Parasternal long-axis M-mode tracings of the left ventricle 


\section{Cellular Physiology Cell Physiol Biochem 2017;42:1313-1325 \begin{tabular}{l|l|l} 
and Biochemistry & $\begin{array}{l}\text { DOI: 10.1159/000478960 } \\
\text { Published } 2017\end{array}$ & $\begin{array}{l}\text { C } 2017 \text { The Author(s). Published by S. Karger AG, Basel } \\
\text { www.karger.com/cpb }\end{array}$
\end{tabular} \\ Zhang et al.: Nobiletin Alleviates Cardiac Hypertrophy In Vivo and Vitro}

(LV) were recorded at the level immediately above the papillary muscles, LV end-diastolic diameter (LVEDd), LV end-systolic diameter (LVESd) and end-diastolic interventricular septal thickness (IVSd) were detected. LV fractional shortening (LVFS) was calculated using the following formula: LVFS(\%) = ( LVEDd - LVESd )/ LVEDd $\times 100$.

In the process of pressure-volume measurements, mice were first anesthetized with $1.5 \%$ isoflurane and maintained their core temperature by a temperature-controlled heating platform, and then a catheter transducer (Millar, Houston, USA) was inserted into the right carotid artery and advanced gradually into the left ventricle. The data was continuously recorded using a Millar Pressure-Volume system (Millar, Houston, USA), hemodynamic parameters including end-systolic pressure (ESP), end-diastolic pressure (EDP), the maximal rate of pressure development (dp/dt max) and minimal rate of pressure decay (dp/dt min) were measured by PVAN data analysis software (Millar, Houston, USA.).

\section{Histological analysis}

Cardiac tissues were fixed in $4 \%$ paraformaldehyde and paraffin-embedded, then cut into $5 \mu \mathrm{m}$ sections. To evaluate the myocyte size, the slides were stained with hematoxylin and eosin (HE) and fluorescein isothiocyanate-labeled wheat germ agglutinin (WGA) (Invitrogen, Carlsbad, USA). WGA staining was performed to measure the accurate myocyte cross-sectional area and 4,6-diamidino-2-phenyl-indole (DAPI) (Invitrogen, Carlsbad, USA) to detect nuclei. Picrosirius red staining was performed to assess the extent of heart fibrosis, and the slides were then visualized using light microscopy and a Nikon PhotoImaging System (H550L, Tokyo, Japan). The cross-sectional areas of the myocardium and cardiac fibrosis were measured using Image-pro Plus 6.0 (Maryland, USA).

Immunohistochemistry for myocardial 4-hydroxynonenal (4-HNE) was conducted, and the sections were deparaffinized and blocked with $8 \%$ goat serum in PBS, followed by incubation with an anti-4-HNE antibody (Abcam, Cambridge, MA) at 1:100 dilution for $12 \mathrm{~h}$ at $4^{\circ} \mathrm{C}$. Thereafter, the sections were incubated with anti-rabbit HRP reagent (Gene Tech, Shanghai, China) for $1 \mathrm{~h}$ at room temperature and developed using a peroxide-based substrate DAB kit (Gene Tech, Shanghai, China). Finally, the sections were dehydrated in ethanol and cleared in xylene. Slides were examined under light microscopy and a Nikon Photo-Imaging System (H550L, Tokyo, Japan).

\section{Neonatal rat cardiomyocyte (NRCM) culture and treatment}

NRCMs were isolated from 1- to 2-day-old Sprague-Dawley rats as previously described [16]. NRCMs were plated onto gelatin-coated culture dishes in DMEM/F12 medium containing 15\% fetal calf serum. After 48 hours, the medium was exchanged for serum-free DMEM/F12, and NRCMs were subsequently treated with $100 \mu \mathrm{mol} / \mathrm{L}$ phenylephrine (PE) (Sigma, Milwaukee, USA) or phosphate buffered saline (PBS) containing $0.1 \%$ bovine serum albumin and/or NOB $(100 \mu \mathrm{mol} / \mathrm{L})$ for 48 hours. NRCMs were transfected with NOX2 siRNA (Santa Cruz Biotechnology, Santa Cruz, CA) or scrambled siRNA (Santa Cruz Biotechnology, Santa Cruz, CA) according to the manufacturer's protocol and then incubated with PE (100 $\mu \mathrm{mol} / \mathrm{L})$ or PBS for 24 hours.

For analysis of the, NRCMs were cultured on coverslips and fixed with $4 \%$ formaldehyde, permeabilized with $0.3 \%$ Triton X-100 in PBS and blocked with $8 \%$ goat serum solution at room temperature. The cells were subsequently stained with a primary antibody against $\alpha$-actinin (Abcam, Cambridge, MA) overnight at $4^{\circ} \mathrm{C}$ and then incubated with a fluorescent secondary antibody. The cell surface area of NRCMs was analyzed using Image-Pro Plus 6.0 software (Maryland, USA).

\section{Evaluation of apoptosis}

Cardiac tissue slides were stained with an Apoptosis Detection Kit (Millipore, Temecula, CA) according to the manufacturer's instructions. Briefly, the sections were initially deparaffinized and pretreated with proteinase $\mathrm{K}(20 \mu \mathrm{g} / \mathrm{ml})$ and then incubated with fluorescein-labeled dUTP. Finally, DAPI (Invitrogen, Carlsbad, CA, USA) was applied to identify all nuclei on the section. The slides were viewed using fluorescence microscopy (BX51, Olympus, Japan) and measured using Image-Pro Plus 6.0 software (Maryland, USA).

\section{Determination of oxidative stress}

To determine the superoxide dismutase 1 (SOD1) concentration of heart tissues, the mice were sacrificed, and their hearts were washed with ice-cold PBS. Cardiac tissues were ground into homogenates and centrifuged at $3,000 \mathrm{~g}$ for $15 \mathrm{~min}$ at $4^{\circ} \mathrm{C}$ to collect the supernatant. The concentration of SOD1 was 


\section{Cellular Physiology Cell Physiol Biochem 2017;42:1313-1325 \begin{tabular}{l|l} 
and Biochemistry Published onlıne: July 12, 2017 & $\begin{array}{l}\text { (c) } 2017 \text { The Author(s). Published by S. Karger AG, Basel } \\
\text { www.karger.com/cpb }\end{array}$
\end{tabular}

determined using commercially available kits purchased from Beyotime Institute of Biotechnology (Haimen, China).

Intracellular ROS were detected using the reactive oxygen species assay kit (Beyotime, Haimen, China) according to the manufacturer's protocols. Briefly, NRCMs were seeded onto 96-well plates and incubated with $10 \mu \mathrm{mol} / \mathrm{L} \mathrm{DCFH-DA}$ probe $(100 \mu \mathrm{L} /$ well $)$ for $30 \mathrm{~min}$ at $37^{\circ} \mathrm{C}$, and the plate was then washed three times with PBS to eliminate residual probe. The fluorescence intensity at $488 \mathrm{~nm}$ excitation wavelength and $525 \mathrm{~nm}$ emission wavelength was determined using a luminometer (Synergy HT, BioTek, USA).

Quantitative real-time reverse transcriptase-polymerase chain reaction (RT-PCR) and western blotting analysis

Cardiac tissues were dissected, immediately frozen and stored in liquid nitrogen. For total RNA extraction from the left ventricles and cultured NRCMs, hearts and NRCMs were thawed in TRIzol reagent (Invitrogen, Carlsbad, CA, USA), and first strand cDNA was synthesized by reverse-transcription of $2 \mu \mathrm{g}$ of total RNA using oligo (dT) primers and a cDNA synthesis kit (Roche, Mannheim, Germany). The mRNA expression level was quantified using a LightCycler 480 SYBR Green Master Mix (Roche, Mannheim, Germany) and normalized against glyceraldehyde 3-phosphate dehydrogenase (GAPDH) mRNA levels. All primers utilized for amplification are shown in Table 1.

For western blotting analysis, left ventricular tissues and cultured NRCMs were homogenized in RIPA lysis buffer (containing $50 \mathrm{mmol} / \mathrm{L}$ Tris-HCl, $0.1 \%$ sodium deoxycholate, $1 \%$ Triton X-100, 5 mmol/L EDTA, $5 \mathrm{mmol} / \mathrm{L}$ EGTA, $150 \mathrm{mmol} / \mathrm{L} \mathrm{NaCl}, 40 \mathrm{mmol} / \mathrm{L} \mathrm{NaF}, 2.175 \mathrm{mmol} / \mathrm{L}$ sodium orthovanadate, $0.1 \%$ SDS, 0.1 $\%$ aprotinin, and $1 \mathrm{mmol} / \mathrm{L}$ phenylmethylsulfonyl fluoride, $\mathrm{pH}=7.2$ ). Subsequently, the homogenates was centrifuged at $12,000 \mathrm{~g}$ for $30 \mathrm{~min}$ at $4^{\circ} \mathrm{C}$ and the supernatant was collected as protein extracts. The protein concentration was examined using a BCA protein assay kit (Thermo, Waltham, MA, USA). Fifty micrograms of protein extract was separated by $10 \%$ SDS-PAGE gel and transferred onto polyvinylidene difluoride (PVDF) membranes (Millipore, Billerica, MA, USA). The PVDF membranes were blocked in Tris-buffered saline containing $5 \%$ skim milk powder for $60 \mathrm{~min}$ at room temperature. The blots were then incubated with the following primary antibodies: rabbit polyclonal anti-caspase-12 and rabbit monoclonal ani-NOX2 and anti-NOX4 (Abcam, Cambrige, MA), rabbit polyclonal anti-GRP78 and mouse monoclonal anti-CHOP (Santa Cruz Biotechnology, Santa Cruz, CA), rabbit anti-GAPDH monoclonal antibody (Abcam, Cambridge, MA). The membranes were scanned using a two-color infrared imaging system (Odyssey; LI-COR Biosciences, Lincoln, NE, USA). Each sample was normalized against GAPDH protein levels.

\section{Statistical analysis}

All data are presented as the mean \pm SD. Statistical comparisons among multiple groups were examined using one-way ANOVA followed by Tukey's post hoc test, and comparisons between two groups were determined using Student's t-test. Statistical analysis was performed using SPSS 17.0 software (SPSS Inc., Chicago, USA). A difference of $P<0.05$ was considered significant.

\section{Results}

NOB supplement mitigated cardiac hypertrophy and reduced fibrosis

The chemical structure of NOB is shown in Fig. 1A. To confirm the protective role of NOB in cardiac hypertrophy, we evaluated

Table 1. Primer sequences used in this study

\begin{tabular}{|c|c|c|c|}
\hline Species & Target genes & & Sequences $\left(5^{\prime}-3^{\prime}\right)$ \\
\hline \multirow[t]{2}{*}{ Rat } & ANP & Sense & ATGGGCTCCTTCTCCATCAC \\
\hline & & Antisense & TCTTCGGTACCGGAAGCTG \\
\hline \multirow[t]{2}{*}{ Rat } & NOX2 & Sense & CAATTCACACCATTGCACATC \\
\hline & & Antisense & CGAGTCACAGCCACATACAG \\
\hline \multirow[t]{2}{*}{ Rat } & NOX4 & Sense & TCCATCAAGCCAAGATTCTGAG \\
\hline & & Antisense & GGTTTCCAGTCATCCAGTAGAG \\
\hline \multirow[t]{2}{*}{ Rat } & GRP78 & Sense & GATAATCAGCCCACCGTAA \\
\hline & & Antisense & TCCTGTCCCTTTGTCTTCA \\
\hline \multirow[t]{2}{*}{ Rat } & CHOP & Sense & ССТTCACTACTCTTGACCCTG \\
\hline & & Antisense & GACCACTCTGTTTCCGTTTC \\
\hline \multirow[t]{2}{*}{ Rat } & GAPDH & Sense & GACATGCCGCCTGGAGAAAC \\
\hline & & Antisense & AGCCCAGGATGCCCTTTAGT \\
\hline \multirow[t]{2}{*}{ Mice } & ANP & Sense & ACCTGCTAGACCACCTGGAG \\
\hline & & Antisense & CCTTGGCTGTTATCTTCGGTACCGG \\
\hline \multirow[t]{2}{*}{ Mice } & BNP & Sense & GAGGTCACTCCTATCCTCTGG \\
\hline & & Antisense & GCCATTTCCTCCGACTTTTCTC \\
\hline \multirow[t]{2}{*}{ Mice } & NOX2 & Sense & GACCATTGCAAGTGAACACCC \\
\hline & & Antisense & AAATGAAGTGGACTCCACGCG \\
\hline \multirow[t]{2}{*}{ Mice } & NOX4 & Sense & TTCСАТССССАAATGCAAAG \\
\hline & & Antisense & TCAGATGCCCTAAAACCGGAG \\
\hline \multirow[t]{2}{*}{ Mice } & GAPDH & Sense & АСТССАСТСАСGGCAAATTC \\
\hline & & Antisense & TCTCCATGGTGGTGAAGACA \\
\hline
\end{tabular}




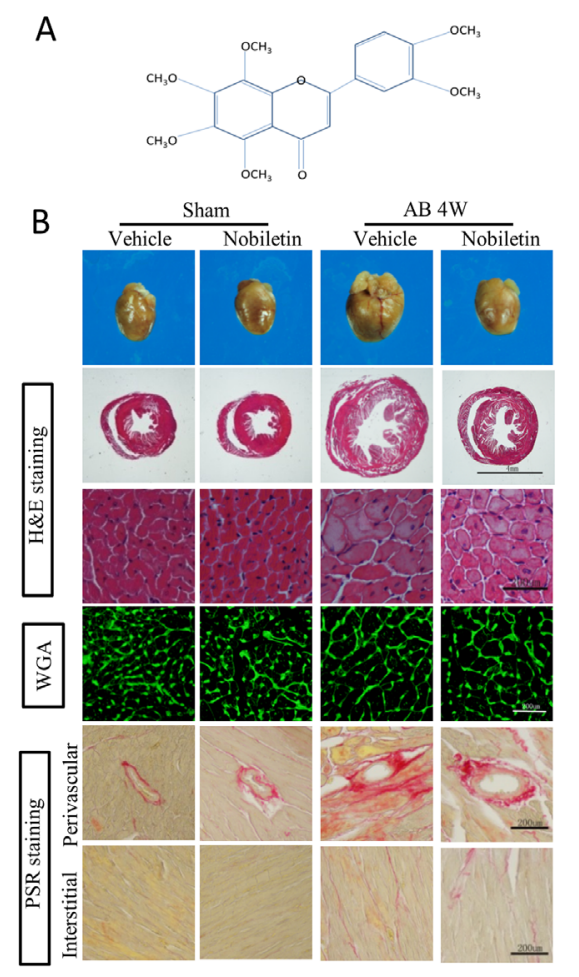

C

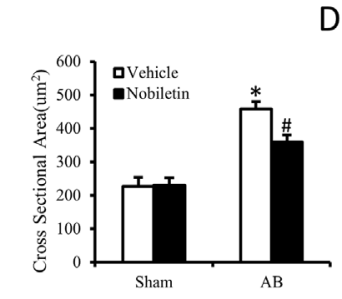

D

E
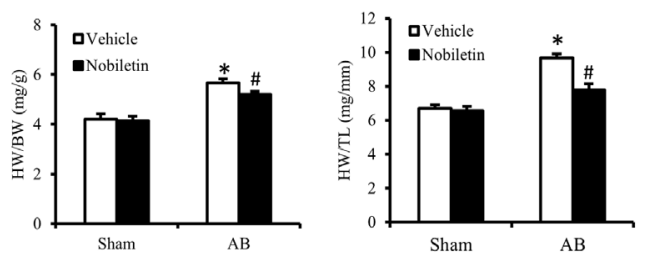

$\mathrm{F}$

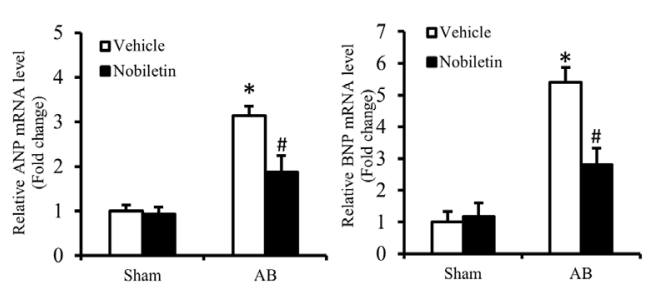

Fig. 1. Effect of treatment with nobiletin (NOB) on aortic banding (AB)-induced cardiac hypertrophy in mice. (A). Chemical structure of NOB. (B). Representative images of the morphological analysis of cardiac hypertrophy as reflected by the whole heart and heart short-axis cross-section (top), hematoxylin and eosin (H\&E) staining (middle) and fluorescein isothiocyanate-labeled wheat germ agglutinin (WGA) staining (middle), picrosirius red (PSR) staining (bottom). (C). Statistical data of the cardiomyocyte cross-sectional areas ( $\mathrm{n}=5$ mice per group; at least 100 cells were measured per mouse). (D). LV collagen ratio of interstitial fibrosis ( $\mathrm{n}=5$ mice per group; at least 5 areas were measured per mouse). (E). Statistical results of heart weight/body weight (HW/BW) and HW/ tibia length (HW/TL) ( $\mathrm{n}=6$ mice per group). (F). Hypertrophic marker natriuretic peptide (ANP) and brain natriuretic peptide (BNP) detected by real-time polymerase chain reaction ( $\mathrm{n}=6$ per group). ${ }^{*} P<0.05$ compared with the sham group, and ${ }^{*} P<0.05$ compared with VEH/ $\mathrm{AB}$.

the morphological change in hearts and mRNA expression of hypertrophic biomarkers. After 4 weeks of $\mathrm{AB}$ surgery, the ameliorated hypertrophic response in NOB/AB mice was further confirmed by morphological examination (Fig. 1B). NOB treatment greatly alleviated the cardiomyocyte hypertrophy and extracellular matrix fibrosis in response to pressure overload, as evidenced by a smaller myocyte cross-sectional area and reduced collagen ratio (Fig. 1C, D). In addition, heart weight/body weight (HW/BW) and HW/tibia length (HW/TL) ratios were dramatically lower in NOB/AB mice compared to VEH/AB mice (Fig. $1 \mathrm{E})$. Consistent with these findings, the mRNA expression of the fetal genes ANP and BNP induced by $\mathrm{AB}$ was markedly attenuated following administration of NOB for 4 weeks (Fig. $1 \mathrm{~F})$. However, there were no significant differences between the VEH/SH and NOB/SH group.

\section{Cardiac function was improved by administration of $N O B$}

To examine whether NOB supplementation alleviated pressure-overload-induced cardiac dysfunction, we assessed cardiac dimension and function by echocardiography (Fig. 2A). NOB treatment resulted in an alleviation of cardiac dysfunction, as reflected by a reduction of LVEDd, LVESd and IVSd (Fig. 2B). Indeed, NOB treatment enhanced systolic function after $\mathrm{AB}$ operation, as indicated by an increase in LV fractional shorting (FS) (Fig. 
A
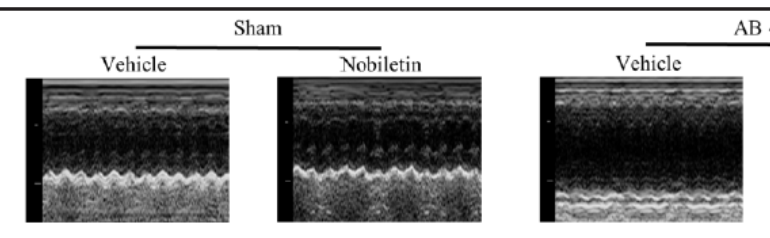

$\mathrm{AB} 4 \mathrm{~W}$

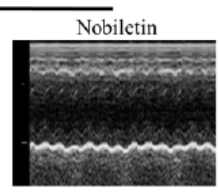

$\mathrm{B}_{6}$ g vehicle
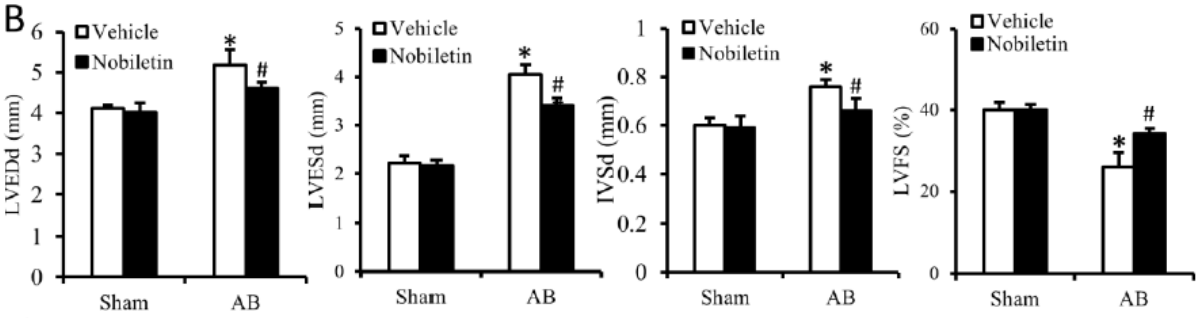

C

Hemodynamic parameters in mice after 4 weeks surgery.

\begin{tabular}{ccccc}
\hline \multirow{2}{*}{ parameter } & \multicolumn{2}{c}{ Sham } & \multicolumn{2}{c}{ AB } \\
\cline { 2 - 5 } & Vehicle $(\mathrm{n}=6)$ & Nobiletin $(\mathrm{n}=6)$ & Vehicle $(\mathrm{n}=6)$ & Nobiletin $(\mathrm{n}=6)$ \\
\hline HR $\left(\mathrm{min}^{-1}\right)$ & $453 \pm 36$ & $460 \pm 40$ & $469 \pm 24$ & $479 \pm 46$ \\
ESP $(\mathrm{mmHg})$ & $106.1 \pm 4.2$ & $108.4 \pm 7.3$ & $133.5 \pm 8.0^{*}$ & $142.1 \pm 4.8^{*}$ \\
EDP $(\mathrm{mmHg})$ & $11.5 \pm 2.1$ & $12.2 \pm 3.0$ & $16.4 \pm 1.9^{*}$ & $14.5 \pm 2.4$ \\
$\mathrm{dp} / \mathrm{dt} \max (\mathrm{mmHg} / \mathrm{s})$ & $8148 \pm 520$ & $7975 \pm 427$ & $5314 \pm 400^{*}$ & $6467 \pm 413^{*}$ \\
$\mathrm{dp} / \mathrm{dt} \min (\mathrm{mmHg} / \mathrm{s})$ & $-8058 \pm 624$ & $-8251 \pm 391$ & $-5100 \pm 459^{*}$ & $-7027 \pm 233^{*}$ \\
\hline
\end{tabular}

$\mathrm{HR}$, heart rate; ESP, end-systolic pressure; EDP, end-diastolic pressure; dp/dt max, maximal rate of pressure development; dp/dt min, maximal rate of pressure decay; ${ }^{*} P<0.05$ compared with Sham group. ${ }^{\sharp} P<0.05$ compare with $\mathrm{VEH} / \mathrm{AB}$.

Fig. 2. Effects of treatment with vehicle or NOB on heart function of cardiac hypertrophy. (A). Representative echocardiographic images of each group. (B). Echocardiographic parameters including left ventricle end-diastolic diameter (LVEDd), left ventricle end-systolic diameter (LVESd), end-diastolic interventricular septal thickness (IVSd) and left ventricle fractional shortening (FS) of each group following AB or sham surgery for 4 weeks. ( $n=6$ mice per group) (C) Hemodynamic parameters in mice after 4 weeks surgery. ( $n=6$ mice per group) $* P<0.05$ compared with the sham group, and ${ }^{\#} P<0.05$ compared with VEH/AB.

2B). Invasive hemodynamic results confirmed preserved systolic ( $\mathrm{dp} / \mathrm{dt}$ max) and diastolic function (dp/dt min) in the NOB/AB mice following 4 weeks pressure-overload (Fig. 2C). Meanwhile, heart rate (HR), end-systolic pressure (ESP) and end-diastolic pressure (EDP) were similar in Sham and AB groups, excluding the possibility that NOB administration influences the pathogenesis of cardiac hypertrophy by affecting these parameters (Fig. 2C). However, we did not observe the statistical differences in cardiac function between the VEH/ SH and NOB/SH mice.

\section{NOB treatment reduced oxidative stress induced by pressure overload}

Increased oxidative stress aggravated cardiac hypertrophy, there was decreased SOD1concentration in the heart tissues following AB for 4 weeks. The activity of SOD1 in cardiac tissues was promoted by NOB supplementation following long-term pressure overload induced by AB (Fig. 3A). To detect the expression of NOX induced by AB surgery, we found increased mRNA and protein expression of NOX2 and NOX4. Moreover, NOB supplementation suppressed the increases in NOX2 and NOX4 expression in NOB/AB mice (Fig. 3B, C, D). Furthermore, Fig. 3E shows that myocardial levels of 4-HNE, another marker of oxidative stress, was mainly in the cytosol of the cardiomyocytes in cardiac tissues after $\mathrm{AB}$ operation, and the staining intensity of 4-HNE was lower in the myocardium of NOB/AB compared with VEH/AB mice (Fig. 3F). The cardioprotective effect exerted by NOB might be 


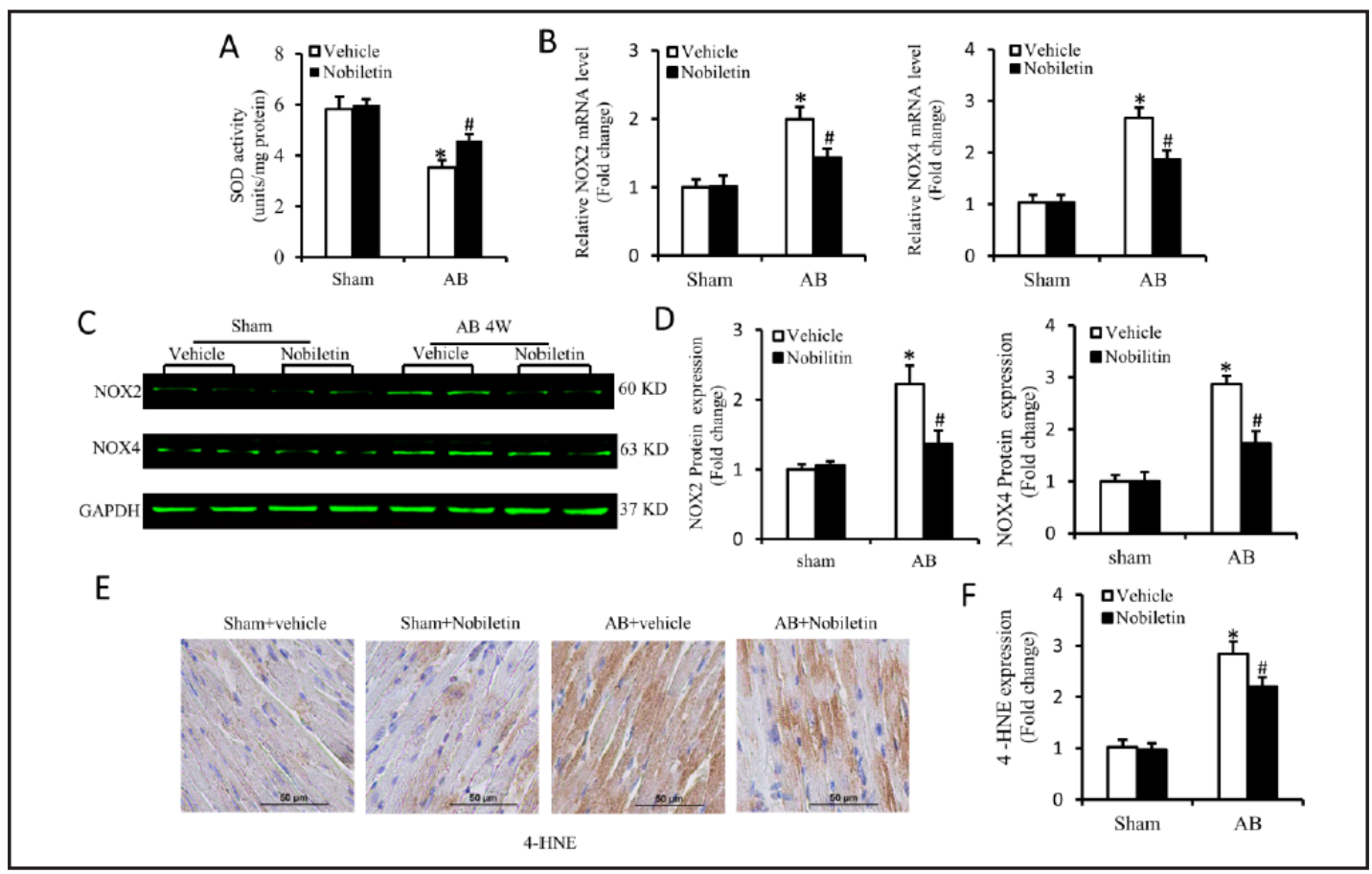

Fig. 3. NOB treatment attenuates pressure-overload-induced oxidative stress. (A) Activity of superoxide dismutase 1 (SOD1). (B) mRNA expression of NOX2 and NOX4 was detected using RT-PCR(n=6 per group). (C) Western blotting analysis of NOX2 and NOX4 levels and (D) quantitative results of NOX2 and NOX4 protein expression (n=6 per group). (E) 4-HNE immunohistochemistry staining in the murine hearts and (F) quantitative results of 4-HNE showing increased oxidative stress ( $\mathrm{n}=5$ mice per group; at least 5 areas were measured per mouse). ${ }^{*} P<0.05$ compared with the sham group, and ${ }^{\#} P<0.05$ compared with VEH/AB.

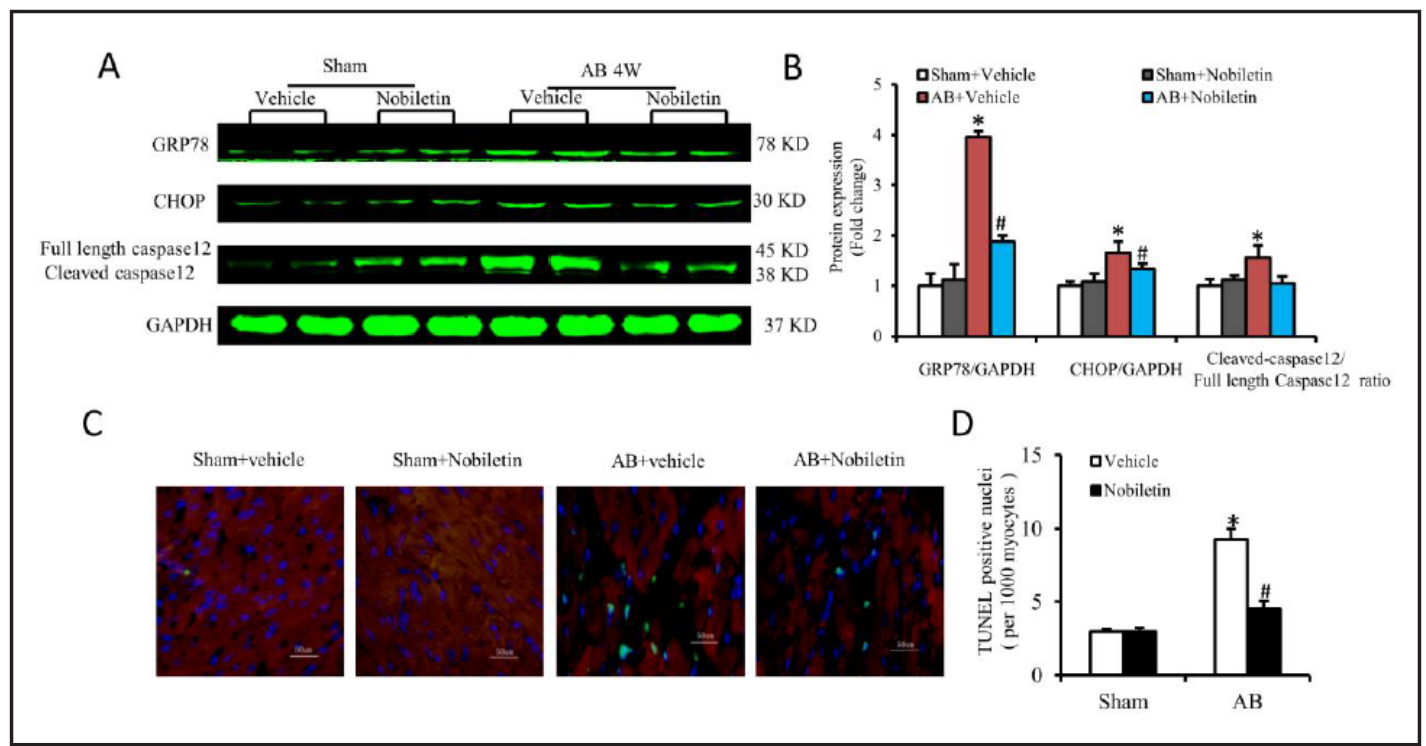

Fig. 4. Increased endoplasmic reticulum (ER) stress and cardiac apoptosis are reduced in advanced pressure-overloaded hearts after NOB treatment. (A) Western blotting analysis of GRP78, CHOP and cleaved-caspase 12/full length caspase12 ( $\mathrm{n}=6$ per group) and (B) quantitative results of GRP78, CHOP and cleaved-caspase 12 /full length caspase 12 ratio. Representative images of cardiac TUNEL staining in cardiac tissues are shown in (C) (blue: normal nuclear; green: TUNEL nuclear). (D) Quantitative results of TUNEL staining. ${ }^{*} P<0.05$ compared with the sham group, and ${ }^{\#} P<0.05$ compared with VEH/AB.

\section{KARGER}


A

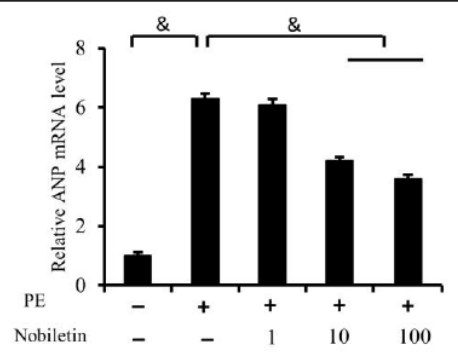

C

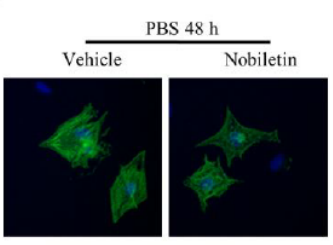

$\mathrm{E}$

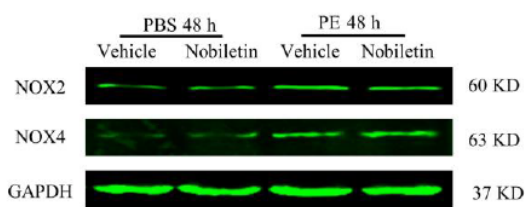

G

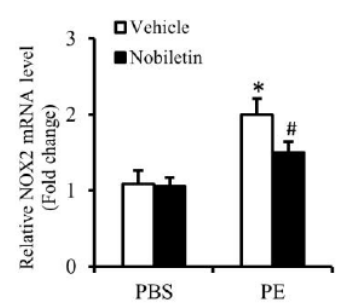

B

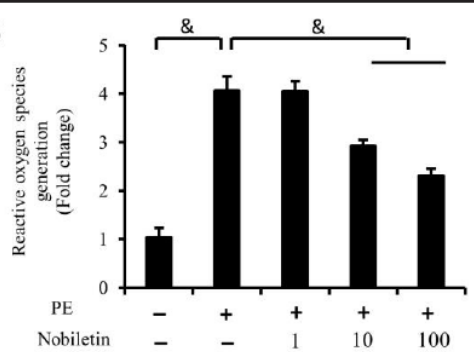

D

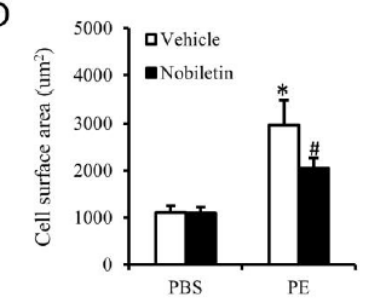

$\mathrm{F} \quad 5, \begin{array}{ll}\text { QPBSS+nobiletin } \\ \text { QPPE }\end{array}$

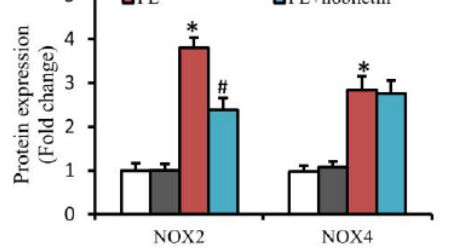

$\mathrm{H}$

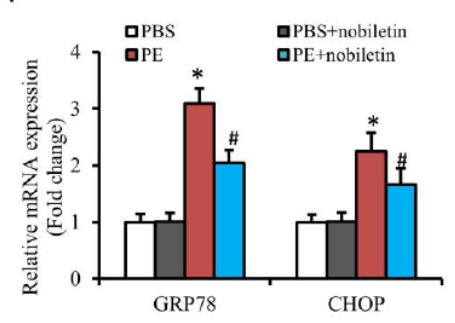

Fig. 5. NOB alleviates phenylephrine (PE)-induced neonatal rat cardiomyocyte (NRCM) hypertrophy in vitro. (A) Effect of NOB on cardiomyocyte hypertrophy induced by PE, while NOB $(100 \mu \mathrm{mol} / \mathrm{L})$ markedly inhibited ANP mRNA expression ( $\mathrm{n}=6$ per group). (B) Generation of reactive oxygen species was blunted by NOB treatment. Representative images of cardiomyocyte hypertrophy are shown in (C) (blue: nuclear; green: $\alpha$-actinin; scale bars: 25 um). The cell surface area was evaluated and is presented in (D) (at least 100 cells per group). (E) Western blotting analysis of NOX2 and NOX4 protein expession ( $\mathrm{n}=3$ per group) and (F) quantitative results of NOX2 and NOX4 protein expression. (G) mRNA expression of NADPH oxidase subunits NOX2 and NOX4 was determined using RT-PCR. ( $n=6$ per group) $(\mathrm{H})$ Endoplasmic reticulum stress was assessed by measuring the mRNA expression of GRP78 and CHOP. ( $n=6$ per group) $* P<0.05$ compared with the PBS group, ${ }^{\#} P<0.05$ compared with the $\mathrm{PE}+$ vehicle group, and ${ }^{\circledR} P<0.05$.

associated with inhibition of NOX2 and NOX4 activities. However, there were no significant differences between the VEH/SH and NOB/SH mice.

$N O B$ treatment attenuated the increase in ER stress and reduced cardiomyocyte apoptosis

Several studies have shown that ER stress plays a crucial role in the development of pathological cardiac hypertrophy [17] and that NOB is able to mediate the process of ER stress [18]. ER chaperones GRP78 is crucial for cell survival/apoptosis under stressful conditions, and ER stress induces apoptosis through CHOP- and caspase-12 dependent signaling pathways. Thus, we investigated whether ER stress was alleviated in response to 
A

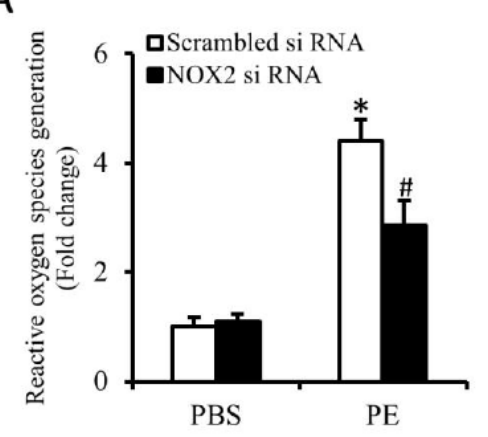

C

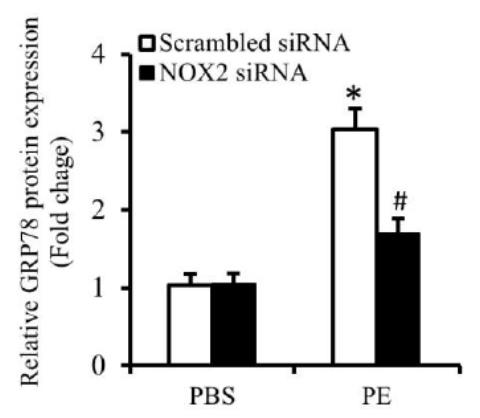

B
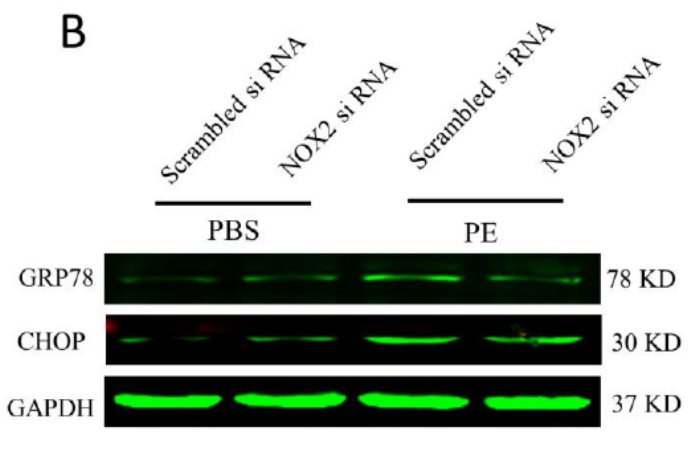

D

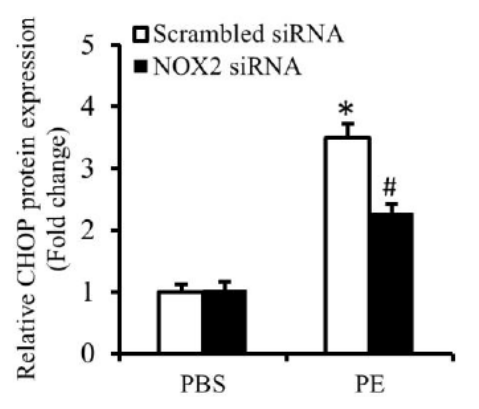

Fig. 6. NOX2 inhibition attenuates PE-induced ROS and ER stress. (A). Effect of NOX2 knockdown on reactive oxygen species generation ( $\mathrm{n}=3$ per group). (B). Knockdown of NOX2 attenuates GRP78 and CHOP protein expression in NRCMs stimulated by PE ( $n=3$ per group). (C, D). Quantitative results of GRP78 and CHOP protein expression. PBS was used as a control. ${ }^{*} P<0.05$ compared with the PBS group, and ${ }^{\#} P<0.05$ compared with the PE + scrambled siRNA group.

NOB supplementation after AB. GRP78, CHOP and cleaved-caspase 12/full length caspase 12 ratio were increased in the tissues of cardiac hypertrophy induced by $A B$, and NOB treatment led to mitigation of ER stress, as reflected by a reduction of GRP78, CHOP and cleaved-caspase 12 protein/full length caspase 12 ratio (Fig. 4A, B). Moreover, Fig. 4C and $4 \mathrm{D}$ revealed that the number of TUNEL-positive nuclei was dramatically increased in the cardiac sections of $A B$ mice compared with sham mice. NOB/AB mice showed a decrease in cardiomyocyte apoptosis, as indicated by a reduced number of TUNEL-positive nuclei (Fig. 4C, D). However, we did not detect any differences between the VEH/SH and NOB/SH mice.

\section{NOB alleviated hypertrophic growth of NRCMs in response to PE stimulation}

The hypertrophic response in NRCMs was measured by the mRNA expression of ANP. As shown in Fig. 5A, NOB could suppress PE-induced cardiomyocyte hypertrophy in a dosedependent manner, and it was confirmed that $100 \mu \mathrm{mol} / \mathrm{L}$ NOB markedly inhibited the hypertrophic response in NRCMs. To determine the anti-oxidative capacity of NOB in NRCMs, the reduction in reactive oxygen species was observed in NOB-treated cells but not in salinetreated cells after stimulation with PE (Fig. 5B). Furthermore, NOB treatment inhibited PEinduced cardiomyocyte hypertrophy, as reflected by a smaller cell surface area (Fig. 5C, D).

We subsequently analyzed whether PE-induced NOX2 and NOX4 protein expression coincided with cardiac hypertrophy in vivo. PE increased NOX2 and NOX4 protein expression (Fig. 5E), however, NOB supplementary markedly decreased NOX2 protein expression and no significant difference in NOX4 expression between NOB-treated cells and saline-treated cells following stimulation with PE (Fig. 5F). RT-PCR analysis was further performed to evaluate the effect of NOB on the mRNA levels of NOX2, NOX4, GRP78 and CHOP in NRCMs stimulated 


\section{Cellular Physiology Cell Physiol Biochem 2017;42:1313-1325 \\ \begin{tabular}{l|l} 
DOI: 10.1159/000478960 & O 2017 The Author(s). Published by S. Karger AG, Basel \\
www.karger.com/cpb
\end{tabular} \\ Zhang et al.: Nobiletin Alleviates Cardiac Hypertrophy In Vivo and Vitro}

by PE. Consistent with the inhibition of NOX2 expression in cardiac hypertrophy in vivo, NOB could also reduce NOX2 mRNA expression induced by PE in NRCMs. Meanwhile, there was no significant difference in NOX4 mRNA expression between NOB-treated cells and salinetreated cells following stimulation with PE (Fig. 5G). Importantly, significant decreases in ER stress, as indicated by a lower mRNA expression of GRP78 and CHOP, were detected in NOBtreated cells but not in saline-treated cells following stimulation with PE (Fig. 5H). Taken together, these results supported the role of NOB as a cardioprotective agent, which could suppress cardiomyocyte hypertrophy and ER stress induced by PE. However, no significance was observed in the PBS-stimulated group.

NADPH oxidase inhibition alleviated PE-induced ROS and ER stress in NRCMS

To further examine the effect of NOX2 on ROS generation, we measured ROS stimulated by PE following transfection with scrambled siRNA or NOX2 siRNA. A reduction in reactive oxygen species was detected in NOX2 siRNA cells but not in scrambled siRNA cells (Fig. 6A). As indicated by the data described above, NOB significantly inhibited the expression of NOX2 in NRCMs stimulated by PE and alleviated ER stress. We hypothesized a direct role of NOX2 on the ER stress. Consistent with this idea, we transfected NOX2 siRNA in NRCMs and then exposed the cells to PE. We found that knockdown of NOX2 dramatically attenuated the increased protein expression of GRP78 and CHOP. (Fig. 6B-D)

\section{Discussion}

The pathophysiology of cardiac hypertrophy is clearly associated with increased oxidative stress. A large body of evidence has demonstrated that ROS generated by NOX is a major source during the progression of cardiac remodeling [3, 4]. Moreover, a flavonoid, NOB, possesses anti-oxidative properties. However, knowledge of the protective effect of NOB on cardiac hypertrophy is still lacking. Here, we found that (1) NOB treatment attenuated cardiac hypertrophy, myocardial fibrosis and LV dysfunction; (2) NOB alleviated increased ER stress and myocyte apoptosis; and (3) NOX2 knockdown in NRCMs mitigated the ER stress induced by PE. Taken together, these findings suggested that NOB may potentially be an ideal therapeutic agent for the treatment of heart failure (Fig. 7).

Under pathological conditions of pressure and/or volume overload, NADPH oxidase becomes overly stimulated, and results in the production of excess oxidants and aggravated oxidative stress [19]. NOX consists of membrane [NOX2 and p22phox] and cytosolic [p47phox, p40 phox, p67 phox] isoforms [20]. NOX2 utilizes NADPH to reduce molecular oxygen to $\mathrm{O}_{2}{ }^{-}$and is expressed in endothelial cells, cardiomyocytes, fibroblasts and vascular smooth muscle cells. Several studies have indicated that NOX2 expression is higher in the infarcted area of human cardiac tissues, and NOX2 knockout mice alleviated post-MI fibrosis and left ventricular dysfunction [21,22]. Additional research studies have revealed that NOX2-deficient mice was less vulnerable to cardiac hypertrophy and fibrosis induced by Ang II infusion. Here, we found that NOB can alleviate cardiac dysfunction and fibrosis induced by pressure overload via inhibition of oxidative stress, a protective mechanism might be partly attributed to a reduced expression of NOX2. The capacity of NOB to alleviate cardiac hypertrophy in vitro was corroborated using NRCMs stimulated by PE, and NOB treatment could inhibit ROS generation and NOX2 expression. Previous data indicated that ROS production mediated by NOX2 was major source in phenylephrine-induced hypertrophy of H9C2 cells [23]. Furthermore, NOB attenuated the PE-induced ROS production in NRCMs, whereas the increased NOX4 mRNA and protein expression observed in NOB-treated NRCMs was not dramatically inhibited.

Another NOX isoform, NOX4, existed in the heart. Furthermore, NOX4 differs from NOX2 such that it is regulated mainly by its expression level and produce $\mathrm{H}_{2} \mathrm{O}_{2}$ via a dismutation of $\mathrm{O}_{2}{ }^{-}$[24]. Global loss of NOX4 contributed to increased susceptibility to pressure-overloadinduced heart failure, which has been linked to defective angiogenesis [25]. However, cardiac- 
Fig. 7. Diagram showed that NOB displayed significant inhibition of NOX2 and NOX4 activity in cardiac hypertrophy. The decrease in oxidative stress caused by inhibition of NOX appeared in parallel with a decrease in ER stress, as well as alleviation in the number of apoptotic cardiomyocytes. Inhibition of oxidative and ER stress by NOB in the myocardium may represent a potential therapy for cardiac hypertrophy.

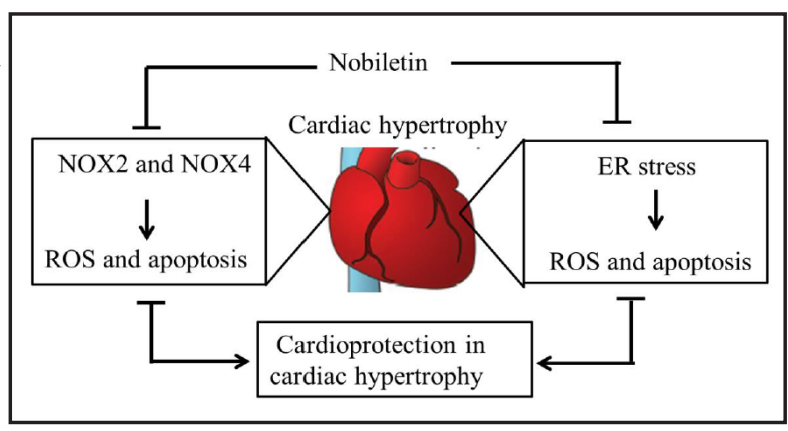

specific deficiency of NOX4 was less vulnerable to mitochondrial and cardiac dysfunction in response to pressure overload [26], and cardiomyocyte-specific loss of NOX4 resulted in increased susceptibility to myocardial ischemic injury in response to energy stress [27]. Taken together, these divergent results may indicate that NOX4 deficiency in cardiomyocytes was beneficial, whereas loss of NOX4 in the vasculature was detrimental and contributed to adaptive angiogenesis. In contrast, NOX2 knockout mice were protected against postMI ventricular dysfunction [22] and showed alleviated cardiac hypertrophy. Collectively, these findings revealed that inhibition of NOX might be an ideal therapeutic target for heart failure. Here, we found that there was a markedly increased mRNA and protein expression of NOX4 induced by AB and that NOB treatment dramatically reduced NOX4 expression after pressure-overload stimulation for 4 weeks.

Various stimuli including ischemia, hypoxia, free-radical exposure could disturb ER homeostasis and cause ER stress. Prolonged ER stress induced by pressure overload was accompanied by the increased expression of GRP78 and CHOP [26, 27]. Moreover, GRP78 was up-regulated under stressful conditions of low oxygen and low calcium. Upon excessive ER stress, GRP78 redistributed to the ER lumen, and cleaved caspase- 12 was released to initiate its activation, which could cause cell death [28]. Previous study indicated that NOB was implicated in ER stress by mediating the expression of GRP78 [18]. In the present study, we found that NOB treatment mitigated ER stress in vivo and in vitro, as indicated by decreased GRP78 expression. The alleviation of ER stress by NOB may result from the inhibition of GRP78. Moreover, ER stress participated in cardiomyocyte apoptosis, and CHOP and caspase-12 were two major signaling pathways involved in ER stress-induced apoptosis. NOX activation aggravated ER stress and involved in pro-apoptotic signaling in the progression of myocardial remodeling [5, 21, 29]. Our results showed NOB supplementation reduced CHOP expression, the ratio of cleaved-caspase 12/full length caspase12 and the number of TUNEL-positive nuclei in NOB/AB mice. The protective effect of NOB may result from the prevention of cardiomyocyte apoptosis via inhibition of NOX, GRP78 and CHOP expression. The capacity of NOB to alleviate oxidative stress in vitro was corroborated using NRCMs stimulated by PE, and NOB treatment could inhibit NOX2 expression but not dramatically blunt NOX4 expression. Inhibition of NOX2 attenuated increased ER stress in the remote non-infarcted tissues after MI [30]. Moreover, NOX2 deficiency protected ER-stressed mice from renal dysfunction [31]. In the present study, we also found that knockdown of NOX2 retarded ER stress in cultured NRCMs after exposure to PE, as indicated by the decreased expression of GRP78 and CHOP, suggesting a direct role of NOX2 in mediating ER stress in cardiomyocytes.

\section{Conclusions}

To the best of our knowledge, this study evaluated the cardioprotective effect of NOB on cardiac hypertrophy induced by AB. We found that NOB displayed significant inhibition of NOX2 and NOX4 expression in vivo but did not markedly reduce NOX4 expression in vitro. The decrease in oxidative stress caused by inhibition of NOX appeared in parallel with a 


\section{Cellular Physiology Cell Physiol Biochem 2017;42:1313-1325 \begin{tabular}{l|l} 
DOI: 10.1159/000478960 & and Biochemistry \\
Published online: July 12, 2017 & $\begin{array}{l}\text { 2017 The Author(s). Published by S. Karger AG, Basel } \\
\text { www.karger.com/cpb }\end{array}$
\end{tabular} \\ Zhang et al.: Nobiletin Alleviates Cardiac Hypertrophy In Vivo and Vitro}

decrease in ER stress, as well as alleviation in the number of apoptotic cardiomyocytes. Moreover, we proposed a direct role of NOX2 in regulating the ER stress-associated protein GRP78 and CHOP. Thus, inhibition of NOX by NOB in the myocardium might be considered a potential therapy for cardiac hypertrophy. However, further investigation in different animal models is under way.

\section{Acknowledgements}

This study was supported by grants obtained from the Key Project of the National Natural Science Foundation (No. 81530012), the National Natural Science Foundation of China (No. 81470516, No.81270303).

\section{Disclosure Statement}

The authors declare that they have no conflicts of interest.

\section{References}

1 Shimizu I, Minamino T: Physiological and pathological cardiac hypertrophy. J Mol Cell Cardiol 2016;97:245.

2 Date MO, Morita T, Yamashita N, Nishida K, Yamaguchi O, Higuchi Y, Hirotani S, Matsumura Y, Hori M, Tada M: The antioxidant N-2-mercaptopropionyl glycine attenuates left ventricular hypertrophy in in vivo murine pressure-overload model. J Am Coll Cardiol 2002;39:907-912.

-3 Zhang M, Perino A, Ghigo A, Hirsch E, Shah AM: NADPH Oxidases in Heart Failure: Poachers or Gamekeepers? Antioxid Redox Signal 2013;18:1024.

$\checkmark 4$ Zhang Y, Tocchetti CG, Krieg T, Moens AL: Oxidative and nitrosative stress in the maintenance of myocardial function. Free Radic Biol Med 2012;53:1531-1540.

-5 Schröder M, Kaufman RJ: Schroder M and Kaufman RJThe Mammalian unfolded protein response. Annu Rev Biochem 2005;74:739-789.

6 Zeeshan HM, Lee GH, Kim HR, Chae HJ: Endoplasmic Reticulum Stress and Associated ROS. Int J Mol Sci 2016;17:327.

7 Logue SE, Cleary P, Saveljeva S, Samali A: New directions in ER stress-induced cell death. Apoptosis 2013;18:537-546.

-8 Li J, Zhu H, Shen E, Li W, Arnold JMO, Peng T: Deficiency of Rac1 Blocks NADPH Oxidase Activation, Inhibits Endoplasmic Reticulum Stress, and Reduces Myocardial Remodeling in a Mouse Model of Type 1 Diabetes. Diabetes 2010;59: 2033-2042.

-9 Runge NRM, Marschall S: Redox signaling in cardiovascular health and disease. Free Radic Biol Med 2013;61:473-501.

10 Murakami A, Nakamura Y, Ohto Y, Yano M, Koshiba T, Koshimizu K, Tokuda H, Nishino H, Ohigashi $\mathrm{H}$ : Suppressive effects of citrus fruits on free radical generation and nobiletin, an anti-inflammatory polymethoxyflavonoid. Biofactors 2000;12: 187-192.

11 Nakajima A, Aoyama Y, Nguyen TTL, Shin EJ, Kim HC, Yamada S, Nakai T, Nagai T, Yokosuka A, Mimaki Y: Nobiletin, a citrus flavonoid, ameliorates cognitive impairment, oxidative burden, and hyperphosphorylation of tau in senescence-accelerated mouse. Behav Brain Res 2013;250:351-360.

-12 Zhang N, Yang Z, Xiang SZ, Jin YG: Nobiletin attenuates cardiac dysfunction, oxidative stress, and inflammatory in streptozotocin: induced diabetic cardiomyopathy. Mol Cell Biochem. 2016;417:87-96.

13 Choi SY, Hwang JH, Ko HC, Park JG, Kim SJ: Nobiletin from citrus fruit peel inhibits the DNA-binding activity of NF-kappaB and ROS production in LPS-activated RAW 264.7 cells. J Ethnopharmacol 2007;113:149-155.

14 Ma ZG, Dai J, Zhang WB, Yuan Y, Liao HH, Zhang N, Bian ZY, Tang QZ: Protection against cardiac hypertrophy by geniposide involves the GLP-1 receptor / AMPK $\alpha$ signalling pathway. Br J Pharmacol 2016;173: 15021516. 


\section{Cellular Physiology Cell Physiol Biochem 2017;42:1313-1325

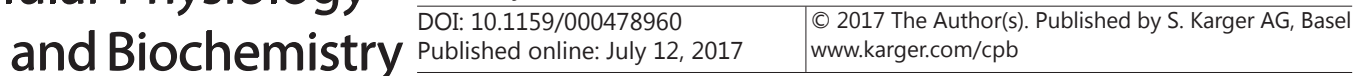 \\ Zhang et al.: Nobiletin Alleviates Cardiac Hypertrophy In Vivo and Vitro}

15 Liu Y, Shen Y, Zhu J, Liu M, Li X, Chen Y, Kong X, Song G, Qian L: Cardiac-Specific PID1 Overexpression Enhances Pressure Overload-Induced Cardiac Hypertrophy in Mice. Cell Physiol Biochem 2015;35:19751985.

16 Jiang DS, Wei X, Zhang XF, Liu Y, Zhang Y, Chen K, Gao L, Zhou H, Zhu XH, Liu PP: IRF8 suppresses pathological cardiac remodelling by inhibiting calcineurin signalling. Nat Commun 2011;5:3303.

17 Sari FR, Widyantoro B, Thandavarayan RA, Harima M, Lakshmanan AP, Zhang S, Muslin AJ, Suzuki K, Kodama M, Watanabe K: Attenuation of CHOP-mediated Myocardial Apoptosis in Pressure-overloaded Dominant Negative p38 $\alpha$ Mitogen-activated Protein Kinase Mice. Cell Physiol Biochem 2011;27:487-496.

18 Moon JY, Cho SK: Nobiletin Induces Protective Autophagy Accompanied by ER-Stress Mediated Apoptosis in Human Gastric Cancer SNU-16 Cells. Molecules 2016;21:914.

19 Streeter J, Thiel W, Brieger K, Jr FJM: Opportunity Nox: The Future of NADPH Oxidases as Therapeutic Targets in Cardiovascular Disease. Cardiovasc Ther 2013;31:125-137.

20 Lassègue B, Martín AS, Griendling KK: Biochemistry, Physiology and Pathophysiology of NADPH Oxidases in the Cardiovascular System. Circ Res 2012;110: 1364-1390.

21 Krijnen PA, Meischl C, Hack CE, Meijer CJ, Visser CA, Roos D, Niessen HW: Increased Nox2 expression in human cardiomyocytes after acute myocardial infarction. J Clin Pathol 2003;56:194-199.

22 Looi YH, Grieve DJ, Siva A, Walker SJ, Anilkumar N, Cave AC, Marber M, Monaghan MJ, Shah AM: Involvement of Nox2 NADPH oxidase in adverse cardiac remodeling after myocardial infarction. Hypertension 2008;51: 319-325.

23 Hahn NE, Musters RJP, Fritz JM, Pagano PJ, Vonk A, Paulus WJ, Rossum ACV, Meischl C, Niessen HWM, Krijnen PAJ: Early NADPH oxidase-2 activation is crucial in phenylephrine-induced hypertrophy of H9c2 cells. Cell Signal 2014;26:1818-1824.

-24 Serrander L, Cartier L, Bedard K, Banfi B, Lardy B, Plastre O, Sienkiewicz A, Fórró L, Schlegel W, Krause KH: NOX4 activity is determined by mRNA levels and reveals a unique pattern of ROS generation. Biochem J 2007;406: 105-114.

-25 Zhang M, Brewer AC, Schröder K, Santos CXC, Grieve DJ, Wang M, Anilkumar N, Yu B, Dong X, Walker SJ: NADPH oxidase-4 mediates protection against chronic load-induced stress in mouse hearts by enhancing angiogenesis. Proc Natl Acad Sci USA 2010;107:18121-18126.

26 Okada K, Minamino T, Tsukamoto Y, Liao Y, Tsukamoto O, Takashima S, Hirata A, Fujita M, Nagamachi Y, Nakatani T: Prolonged endoplasmic reticulum stress in hypertrophic and failing heart after aortic constriction: possible contribution of endoplasmic reticulum stress to cardiac myocyte apoptosis. Circulation 2004;110:705-712.

27 Sciarretta S, Zhai P, Shao D, Zablocki D, Nagarajan N, Terada LS, Volpe M, Sadoshima J: Activation of NADPH oxidase 4 in the endoplasmic reticulum promotes cardiomyocyte autophagy and survival during energy stress through the protein kinase RNA-activated-like endoplasmic reticulum kinase/eukaryotic initiation factor $2 \alpha$ /activating transcripti. Circ Res 2013;113:1253-1264.

28 Momoi T: Caspases involved in ER stress-mediated cell death. J Chem Neuroanat 2004;28:101-105.

-29 Kuroda J, Ago T, Matsushima S, Zhai P, Schneider MD, Sadoshima J: NADPH oxidase 4 (Nox4) is a major source of oxidative stress in the failing heart. Proc Natl Acad Sci USA 2010;107:15565-15570.

-30 Li B, Tian J, Sun Y, Xu TR, Chi RF, Zhang XL, Hu XL, Zhang YA, Qin FZ, Zhang WF: Activation of NADPH oxidase mediates increased endoplasmic reticulum stress and left ventricular remodeling after myocardial infarction in rabbits. Biochim Biophys Acta 2015;1852:805-815.

-31 Li G, Scull C, Ozcan L, Tabas I: NADPH oxidase links endoplasmic reticulum stress, oxidative stress, and PKR activation to induce apoptosis. J Cell Biol 2010;191:1113-1125. 